\title{
Spontaneous Current Study in Wheat Starch Nano Powder
}

\author{
M.S. GAUR ${ }^{a, *}$ AND K. GAUR ${ }^{b}$ \\ ${ }^{a}$ Department of Physics, Hindustan College of Science and Technology, Farah, Mathura (U.P.), India \\ ${ }^{b}$ Department of Bioscience, Govt. R.D. Girls College, Bharatpur (Rajasthan), India
}

(Received June 21, 2009; in final form March 12, 2010)

\begin{abstract}
Spontaneous current study has been carried out in $70 \mathrm{~nm}$ particle size of wheat starch nano powder. The starch nano powder was prepared by mechanical grinding method. The spontaneous current was found to be induced and flowing in external circuit under short circuit condition by heating a sandwitched system. Sandwitched system consist of a starch nano powder (i.e. powder in different weight ratio of distilled water) between similar $(\mathrm{Al}-\mathrm{Al})$ and dissimilar $(\mathrm{Al}-\mathrm{Zn}, \mathrm{Al}-\mathrm{Cu}, \mathrm{Al}-\mathrm{Ag})$ electrode combination. The Spontaneous current spectra show two peaks lies in $46 \pm 4{ }^{\circ} \mathrm{C}$ and $72 \pm 4{ }^{\circ} \mathrm{C}$, respectively, in the first heating run. However, only single peak was observed in second heating run. In the dissimilar electrode combination the first peak shifts to lower temperature side, while second peak shifts to higher temperature side. Spontaneous current mechanism could be explained on the basis transitional changes of starch nano powder caused by influence of water. The temperature dependent open circuit voltage $V_{\text {oc }}$ was recorded. A linear relationship was observed between dissimilar electrodes system due to difference in electrode work functions. It has been observed that spontaneous current is influenced by water and depends on the choice of electrode material.
\end{abstract}

PACS numbers: 77.22.Ej, 77.22.Cm, 77.22.Jp

\section{Introduction}

In recent years many efforts have been taken to study the thermally stimulated discharge process in insulating synthetic polymers [1-6], but only little attention has been paid towards the biopolymers [7]. The various mechanisms of charge and energy transfer in biopolymers in the part of living biological systems (cellulose, collagen, melanin, etc.) were studied by Glasser [8-10] in detail. The biopolymers have several advantages in general. They are commercially cheaper and non-toxic compared to that of synthetic polymers. Moreover, in many cases the synthetic polymers have toxic effects.

It has been shown that biotechnology is not only applicable to genomic sequencing and clinical diagnosis and treatment, it can also have a major impact on nonbiotech applications, opening up a whole new field for bioengineering. The biopolymers possess unique optical and electromagnetic properties, including low and tunable electrical resistivity, ultralow optical and microwave loss, etc. The organic field effect transistors (OFETs), organic light emitting diodes (OLEDs), and nonlinear optical (NLO) polymer electro-optic (EO) modulators fabricated from new biopolymer have been demonstrated in literature [11-13].

All matter contains electrically charged particles. Most of the materials are electrically neutral because positive and negative charges are present in equal numbers. When the balance of electric charges is disturbed, we experi-

* corresponding author; e-mail: mulayamgaur@rediffmail.com ence electrical effects. Due to this fact the spontaneous current is the basic feature for all living and nonliving matter and plays a significant role in the viability of biological matter. The electrical properties of biopolymers depends on their structure, morphology and processing. Since starch is a high molecular weigh crystalline class of biopolymer made up of two components viz. amylase and amylopection. The amylase is an unbranched chain of 300 to $1000 \alpha$-D glucose with 1-4 glycosidic bonds. The amylopection is also made up of $\alpha$-D glucose unit but it is branched like a tree. The ratio of amylase to amylopection in starch is different for different tissues. The chemical structure of starch is shown in Fig. 1. 1-6 linkage causes the formation of a branching point, which then allows another chain of 1-4 linkages to develop. This 3 -D branching also produces other points at which glucose may be added by condensation or removed by hydrolysis. The wheat starch normally contains about $20 \%$ amylase and $80 \%$ amylopection. The presence of amylase and amylopection gives rise to an internal electric field [14-15]. This effect is used to measure the variation of electrical properties under the influence of external field, temperature and radiation, etc. The dielectric study of starch is reported by several authors [16-18] under different conditions, but mechanism of spontaneous current in starch is still unknown.

The study of electrical properties of starch is an important topic of research because of its importance in drug delivery system. Recently, application of starch nanoparticle cross linked with sodium tripoly phosphate for better stabilization was discussed in details [19-20]. The objective of the present work is to investigate mechanism 


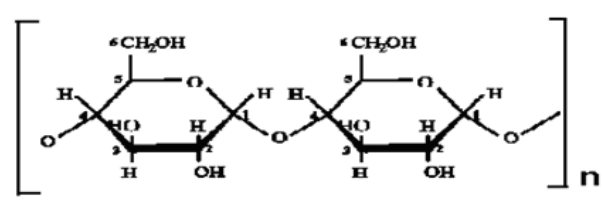

Fig. 1. Chemical structure of starch.

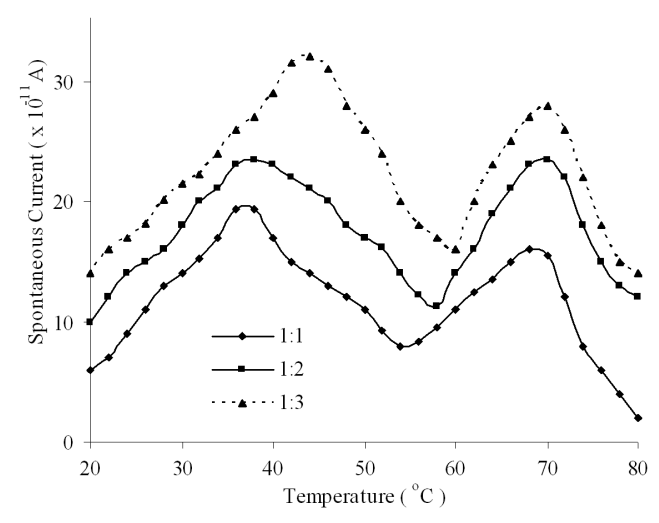

Fig. 2. Spontaneous current spectra of starch in different weight ratios.

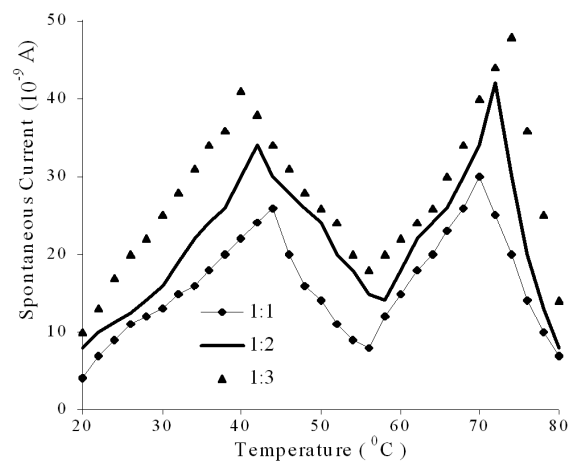

Fig. 3. Spontaneous current spectra of starch nano powder in different weight ratios.

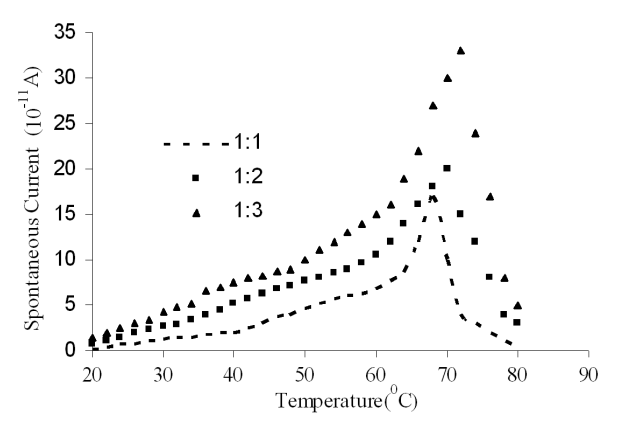

Fig. 4. Spontaneous current spectra of starch nano powder in different weight ratios during second heating run.

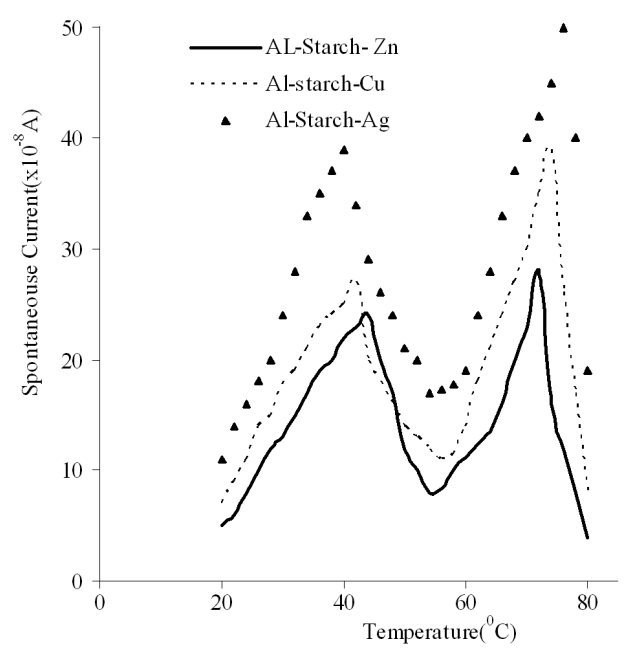

Fig. 5. Spontaneous current spectra of starch nano powder in dissimilar electrode combination.

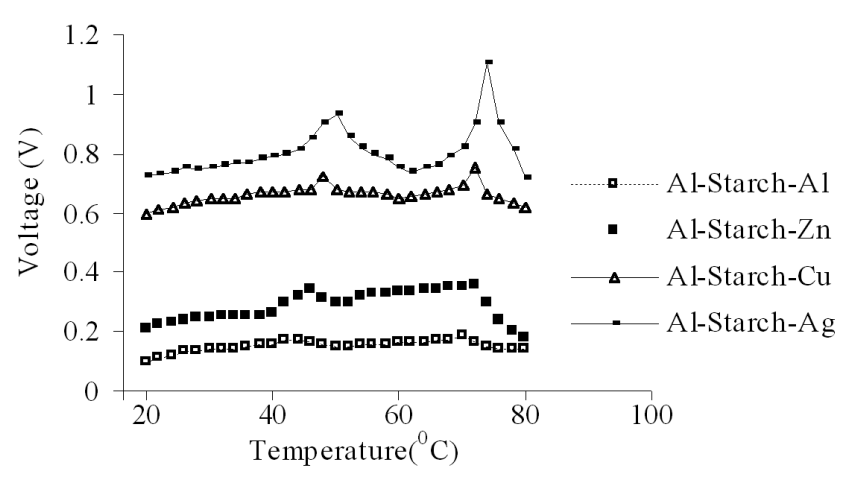

Fig. 6. Open circuit voltage spectra of starch nano powder in dissimilar electrode combination.

of heat induced current in starch nano powder by measuring the spontaneous current and open circuit voltage, which may proved to be more interesting for scientific and technological point of view.

\section{Experimental}

The wheat starch supplied by Merck India Ltd. (i.e. granules size $20-45 \mu \mathrm{m}$ ) is used in present study. The nano powder of wheat starch was prepared by using traditional grinding using pastel and Motor. Total time of continues grinding was $5 \mathrm{~h}$ to prepare $10 \mathrm{gm}$ powder. The particle size was $70 \mathrm{~nm}$ were characterized by XRD. It was observed that spontaneous current was tremendously increased when observation was carried out in nano particles. Our experiments, samples of starch/starch nano powder with different weight ratios were prepared for spontaneous current measurement. The powder was mixed with double-distilled de-ionized water to prepare starch water slurry of $1: 1,1: 2,1: 3$ ratios by weight. All samples were properly stirred mechanically for around 15 minutes before observation. The samples were taken 
on aluminum foil cleaned with ethyl alcohol before using, after that $80 \mu \mathrm{m}$ thick circular Teflon lining with another aluminum electrode was covered over the sample. The sample was immediately put into the heating device. The spontaneous current was recorded by means of sensitive electrometer (Scientific Equipments, Roorkee, India, DPM-111) with the heating rate of $4^{\circ} \mathrm{C} / \mathrm{min}$. The surface potential was measured using microvoltmeter (scientific Roorkee, India). It is found, that the results are reproducible with experimental error of $\pm 10 \%$.

\section{Results and discussion}

Figure 2 shows the spontaneous current spectra of wheat starch of granules size $20-45 \mu \mathrm{m}$ at constant heating rate of $4{ }^{\circ} \mathrm{C} / \mathrm{min}$ with similar $(\mathrm{Al}-\mathrm{Al})$ electrode combination. Each curve shows two peaks at different temperatures. Ideally, biopolymer is invisible for investigation of spontaneous relaxation properties. But if these materials are heated at constant temperature, it produces an electrochemical potential difference between metallic electrode and the biopolymer. This effect is very well observed in case of many polymers by several researchers [21-22].

The order of current for this peak is found to be $10^{-11} \mathrm{~A}$, while study is carried out in starch nano powder in same condition (Fig. 3) gives the two sharp peak and tremendous increase of current (i.e. $\left.10^{-9} \mathrm{~A}\right)$. This behaviuor could be understood in terms of the conductivity. The conductivity of material increases, if the size of particle is reduced upto nano level [23]. The second peak is appeared at higher temperature in all cases. The water molecules are thought to associate with oppositely charge groups in the starch. This combination of charged groups binds more water than the charged groups alone and starch hydration is increased. The starch hydration enhances the generation of ions and has been observed to move from upper electrode to lower electrode (i.e. current flows from lower to upper electrode in the external circuit); however, more and more ions are generated in starch nano powsder. In other hand the first peak is assigned to absorption of water molecules and nonappearance of this peak in second heating run is attributed to gelatinization of starch and reduction of water molecules. The appearance of strong peak in second heating run may confirm the trapping of ionic space charge.

The result of spontaneous current during second heating run is shown in Fig. 4. It shows that current do not have similar order as in first heating run. This indicates that the reduction of current caused by the dehydration of starch samples results shifting of peak towards higher temperature. This is due to the behaviour of bound water as demonstrated in other biopolymers [24-26].

Figure 5 shows the spontaneous current spectra recorded for different combination of electrodes. These spectra consist of two peaks. The order of current was found to be of $10^{-8} \mathrm{~A}$ for dissimilar electrode system. The position of low temperature peak is shifted towards lower temperature side, however, position of second peak is shifted towards higher temperature side by varying the electrode material. The order of current was observed to be lower in similar electrode system, than the dissimilar electrode system.

Figure 6 represents the temperature dependence of open circuit voltage. The open circuit voltage is observed according to work function difference of corresponding electrodes, that shows a linear relationship.

In the starch structure $\mathrm{OH}$ group is at reactive position at a certain temperatures. The active group is responsible for formation of large number of pairs of opposite charges (i.e. $\mathrm{H}^{+}$and $\mathrm{e}^{-}$or $\mathrm{H}^{+}$and $\mathrm{OH}^{-}$). The increase of spontaneous current in starch nano powder is caused by absorption of water molecules form the weak complex with side group. This may be attributed to dissociation of weak complex along with liberation of ions.

The effects of dilutents and plasticizers on $T_{\mathrm{g}}$ of starch can be discussed in the following way:

$$
\frac{1}{T_{\mathrm{g}}}=\frac{M_{(\mathrm{starch})}}{T_{\mathrm{g}(\mathrm{starch})}}+\frac{M_{\left(\mathrm{H}_{2} \mathrm{O}\right)}}{T_{\mathrm{g}\left(\mathrm{H}_{2} \mathrm{O}\right)}},
$$

where $T_{\mathrm{g}\left(\mathrm{H}_{2} \mathrm{O}\right)}$ is the glass transition of water and $M_{\left(\mathrm{H}_{2} \mathrm{O}\right)}$ is its weight fraction. Similarly, $T_{\mathrm{g} \text { (starch) }}$ and $M_{\text {(starch) }}$ have their usual meaning. Therefore, spontaneous current is the water controlled mechanism brought about by absorption of water. The biopolymer like starch which accommodates water molecules and controls the spontaneous current mechanism having maximum rate of emission process at $T_{\mathrm{g}}$. The quasi - equilibrium condition of dissociation of water may alter during heating that causes the movement of charge carriers. The starch chain gets loosened near $T_{\mathrm{g}}$, facilitating the release of charge carriers resulting into a peak.

In other hand spontaneous current behaviour of starch/starch nano powder was also found to depend upon hydration, indicating water structure around it. When sample is heated linearly, intrinsic characteristics of material was obtained between two main bands in the characteristics, as it is shown in Figs. 2, 3, and 5 . Starch is a well-known polar polymer having -OH dipolar group. The low temperature peak is caused by dipolar effect. The activation energy of this peak, lies within 0.21 to $0.41 \mathrm{eV}$, is in agreement with dipolar effect. The low temperature peak is shifted towards higher temperature side due to orientation of - $\mathrm{OH}$ dipole, which is internally hydrogen bonded in the molecules. This bond is known to be weaker for that particular hydroxyl group, and some of these dipoles would be free to orient under the influence of temperature. The high temperature peak was found to be due to ionic space charge. In most of the sources the nature of the space charge is unknown. However, some of the sources are contacting electrodes, protonic carriers and ions.

The charge carriers are generated (i.e. positive/negative) at the metal surface due to an electron/ion transfer between metal and dielectric [27]. On the other hand, existence of weak internal field and electrochemical nature of starch, the electrode starch interfaces may have elec- 
tron/ions due to exchange between them (charged layer). If the net flow of charge carriers is from metal to starch, the metal will suffer a certain depletion of its charge and its surface may require a net opposite charge. Thus, charge carriers present in bulk or near the electrodes are supposed to be mobile for internal and external conduction mechanism.

The open circuit voltage $V_{\text {oc }}$ developed at an electrode seems to be due to the potential difference between electrodes, possible orientation of polar side group and charge exchange between metal and starch due to difference in their work function. In dissimilar electrode system the electron exchange occurs between electrodes dielectric interfaces and for these combinations the work function differences of metals may have a controlling nature of $V_{\mathrm{oc}}$ magnitudes. These results could be explained in terms of net difference of work function of both the metals with the work function of starch. It has been reported [28], that charge injection into the polymers decreases with increase in work function. The work function difference of $\mathrm{Al}-\mathrm{Cu}, \mathrm{Al}-\mathrm{Zn}, \mathrm{Al}-\mathrm{Ag}$ seem to be greater than the work function differences of similar electrode metal system.

\section{Conclusions}

It is concluded that the magnitude of spontaneous current depends upon choice of electrode system and mesh size of starch. The spontaneous current is flowing in external circuit due to induced electromotive force arising from an electrochemical process at the metal starch interface followed by generation of charge carriers.

\section{Acknowledgments}

Author (KG) gratefully acknowledge the financial support granted by University Grant Commission New Delhi, India.

\section{References}

[1] P.K. Khare, K. Devendra K. Sahu, Ashutosh Verma, Rudrakant Srivastava, Indian J. Pure Appl. Phys. 42, 693 (2004).

[2] Electrets, Vol. 1, 3rd Ed. G.M. Sessler, Laplacian Press, Morgan Hill, 1999.

[3] P.K. Khare, J.M. Keller, M.S. Gaur, Ranjeet Singh, S.C. Datt, Polym. Int. 39, 342 (1996).
[4] H. Frensch, J.H. Wendroff, Polymer 27, 1332 (1986).

[5] Electrical Properties of Polymers, Ed. D.A. Seanor, Academic Press, New York 1982.

[6] T. Blythe, D. Bloor, Electrical Properties of Polymers, 2nd Edition, Cambridge University Press, 2005.

[7] S. Chandra, Superionic Solids - Pricipal and Application, Horth-Holland, Amsterdam, 1981.

[8] L. Glasser, Chem. Rev. 75, 21 (1975).

[9] F. Chivrac, E. Pollet, P. Dole, L. Averous, Carbohydrate Polymers 79, 941 (2010).

[10] F. Starzyk, A. Chrzanowska, W. Luzney, M.O. Eniechowski, Arch. Mater. Sc. Eng. 39, 111 (2009).

[11] W Hagen, A. Li, J. Grote, K. Hopkins, Appl. Phys. Lett. 88, 171109 (2006).

[12] E. Heckman, P. Yaney, J. Grote, F. Hopkins, M. Tomczak, Proc. SPIE 6117, 0K1 (2006).

[13] B. Singh, S. Sariciftci, J. Grote, F. Hopkins, J. Appl. Phys. 100, 024514 (2006).

[14] B.F. Salisbury, W.C. Ross, Plant Physiology, 4th ed., Wedsworth Publishing, Belmont (CA) 2000.

[15] Phytochemistry, Ed. D.J. Manner, Van Nostrand Reinhold, New York 1973.

[16] L.A. Miller, J. Jordon, E.A. Davis, Cereal Chem. 68, 441 (1991).

[17] J.W. Donovan, Biopolymers 18, 263 (1979).

[18] R.E. Mugdett, Food Technol. 36, 109 (1982).

[19] R.H. Marchessault, F. Ravenelle, X.X. Zhu, Polysaccharides for Drug Delivery and Pharmaceutical Applications, Oxford University Press, 2006.

[20] C.K. Simi, T. Emilia, Abraham, Bioprocess Biosyst. Eng. 30, 173 (2007).

[21] J. Van Turnhout, Electrets, Ed. G.M. Sessler, Springer-Verlag, Berlin 1980, p. 84.

[22] J. Ahmed, H.S. Ramaswamy, V.G.S. Raghavan, J. Food Engg. 80, 1125 (2007).

[23] A.K. Bandyopadhyay Nano materials, 2nd ed., New Age International, New Delhi 2008.

[24] H. Frölichlich, Int. J. Quantum Chem. 2, 641 (1968).

[25] H. Frölichlich, Phys. Lett. 44A, 385 (1973).

[26] H. Frölichlich, Proc. Natl. Acad. Sci. USA 72, 4211 (1975).

[27] J.O.M. Backris, A.K.N. Reddy, Modern Electrochemistry, Plenum Press, New York 1977, p. 32.

[28] P.K. Khare, M.S. Gaur, A.P. Shrivastava, Indian J. Pure Appl. Phys. 31, 399 (1993). 